\title{
Maximum A Posteriori Adjustment of Adaptive Transversal Filters in Active Noise Control
}

\author{
Iman Tabatabaei Ardekani, Xiao Zhang, Hamid Sharifzadeh and Jari Kaipio
}

\begin{abstract}
This paper develops a novel approach to adaptive active noise control based on the theory of Bayesian estimation. Control system parameters are considered as statistical variables and a formulation for the joint probability density function of them is derived. An optimal solution for the system parameters is then calculated through maximizing the density function. An efficient adaptive algorithm for iterative calculation of the optimal parameters is proposed. It is shown that the wellknown FxLMS algorithm can be derived as a special case of the proposed algorithm, where the noise to be canceled is a white Gaussian process. Simulation results verify the preference of the proposed system to the traditional active noise control systems in terms of steady-state performance and convergence rate. It is also shown that the preference of the proposed system is much more evident when the noise to be canceled is not white. Finally, a successful implementation of the proposed system in an experimental acoustic duct is reported.
\end{abstract}

\section{INTRODUCTION}

Active Noise Control (ANC) relies on the destructive superposition of sound fields [1]. In a generic adaptive ANC system, an adaptive controller (or filter), called ANC filter, is responsible for driving a canceling source that produces an optimal cancelling sound field. ANC filter parameters are estimated in such a way that the superposition of the canceling sound field and the unwanted sound field (noise) at the location of a desired Zone of Silence (ZOS) becomes minimal. Unfortunately, standard adaptive control algorithms [2] cannot estimate ANC filter parameters due to the existence of an unknown signal channel between the canceling source and the desired ZOS. For solving this problem, several ANC specific adaptive algorithms (usually calledadaptive ANC algorithms) such as Filtered-reference Least Mean Square (FxLMS) or Filtered-reference Recursive Lease Square (RLS) algorithms have been derived [3]. The main motivation of this paper is to derive a novel adaptive ANC algorithm based on the theory of Bayesian estimation and more particularly the theory of Bayesian inverse problems [4].

Fig 1 shows the block diagram of a generic ANC system [5], [6]. In this figure, $\xi(n), y(n)$ and $r(n)$ represent noise, cancelling sound and residual noise at ZOS. Considering that the noise source(s) is placed at an arbitrary location, noise waves must travel across an acoustic system (or channel), called the primary path, to reach ZOS. $\xi(n)$ which is the received noise signal at the ZOS cannot be measured as it

Iman Tabatabaei Ardekani, Hamid Sharifzadeh and Xiao Zhang are currently with the Department of Computer Science, Unitec Institute of Technology, New Zealand, e-mail: iardekani@unitec.ac.nz Jari Kaipio and Iman Tabatabaei Ardekani are with the Department of Mathematics, The University of Auckland, Auckland, New Zealand. intends to be combined by the cancelling sound. However, a microphone, called the reference microphone, is placed close to the noise source to measure a reference signal $x(n)$. The control signal $u(n)$ is the response of the ANC filter $W$ to $x(n)$. This signal is converted to sound by a loudspeaker (canceling source) and propagates through an electro-acoustic signal channel, called the secondary path to reach ZOS. The secondary path is shown by $S$ in the block diagram shown in Figure 1. In fact, the cancelling signal $y(n)$ is the response of the secondary path $S$ to the control signal $u(n)$. Acoustic signals $\xi(n)$ and $y(n)$ combine with each other at ZOS, resulting in residual noise signal $r(n)$. A microphone, called the error microphone, is placed at ZOS to measure $r(n)$.

Adaptive ANC algorithms such as Filtered-x Least Mean Square (FxLMS) algorithm [7], [8] are responsible for the adaptive adjustment of the ANC filter $W$ subject to the minimization of the residual noise $r(n)$. Like FxLMS algorithm, most of adaptive ANC algorithms require an estimate model for the secondary path $S$. This model is obtained by using secondary path modeling techniques [9]-[12], which is an essential part of ANC systems. FxLMS is a very computationally efficient algorithm but it suffers from slow convergence rate. Furthermore, its stability is very sensitive to the parameters of the noise, media and control system [13]-[15].

In this paper, a novel algorithm for adaptive adjustment of the ANC filter based on the theory of Bayesian estimation [4] is developed. This method was previously used by the authors to develop a novel solution for on-line secondary path modeling [12]. However, it has not been used for solving the main ANC problem that is the adaptive adjustment of the ANC filter. The rest of the paper is organized as follows. Section 2 develops a novel approach to adaptive ANC based on the theory of Bayesian estimation, resulting in a formulation for an optimal ANC filter. Section 3 proposes a novel algorithm for the iterative estimation of the proposed optimal ANC filter. Section 4 discusses computer simulation results. Section 5 reports a successful implementation of the proposed algorithm and finally section 6 gives concluding remarks.

\section{BAYESIAN APPROACH TO ADAPTIVE ANC}

\section{A. Mathematical modeling of a generic ANC system}

In a generic ANC system, residual noise $r(n)$ can be formulated as the superposition of the original noise $\xi(n)$ and cancelling sound $y(n)$ :

$$
r(n)=\xi(n)+y(n)
$$

Referring to Figure 1, $y(n)$ is the response of the secondary path $S$ to the control signal $u(n)$. The adaptive filter $W$ 
is responsible for producing the control signal $u(n)$ from a reference signal $x(n)$. Usually, the adaptive filter used in ANC has a transversal structure of length $L$ and the secondary path is a linear system with a finite-duration impulse response of length $Q$. In this case, the impulse response coefficients of the ANC filter and secondary path can be represented by the following vectors.

$$
\begin{aligned}
\mathbf{w} & =\left[\begin{array}{llll}
w_{0} & w_{1} & \ldots & w_{L-1}
\end{array}\right]^{T} \\
\mathbf{s} & =\left[\begin{array}{llll}
s_{0} & s_{1} & \ldots & s_{Q-1}
\end{array}\right]^{T}
\end{aligned}
$$

From the block diagram given in Figure 1, $y(n)$ can be expressed by

$$
y(n)=\sum_{q=0}^{Q-1} s_{q} u(n-q)
$$

Similarly, $u(n)$ can be expressed by

$$
u(n)=\sum_{l=0}^{L-1} w_{l} x(n-l)
$$

Combining Eqs. (4) and (5) results in

$$
y(n)=\sum_{q=0}^{Q-1} \sum_{l=0}^{L-1} s_{q} w_{l} x(n-l-q)
$$

Eq. (6) can be re-expressed by

$$
y(n)=\sum_{l=0}^{L-1} w_{l} x_{s}(n-l)
$$

where $x_{s}(n)$, called the filtered-reference signal is given by

$$
x_{s}(n)=\sum_{q=0}^{Q-1} s_{q} x(n-q)
$$

Substituting Eq. (7) into (1) results in the following formulation for the residual noise.

$$
r(n)=\xi(n)+\sum_{l=0}^{L-1} w_{l} x_{s}(n-l)
$$

The optimization problem of adaptive ANC is to find the coefficients $w_{l}$ subject to the minimization of an appropriate cost function of $r(n)$.

\section{B. Proposed optimization method}

Let us form a measurement vector $\mathbf{r}$ by using the most recent samples of the residual noise $r(n)$ :

$$
\mathbf{r}=\left[\begin{array}{llll}
r(n) & r(n-1) & \ldots & r(n-M+1)
\end{array}\right]^{T}
$$

where $M$ is the length of the measurement vector. From Eq.(1), $\mathbf{r}$ can be formulated by

$$
\mathbf{r}=\boldsymbol{\xi}+\mathbf{y}
$$

where the noise vector $\boldsymbol{\xi}$ and the cancelling sound vector $\mathbf{y}$ are formed by samples of the noise and cancelling sound signals:

$$
\boldsymbol{\xi}=\left[\begin{array}{llll}
\xi(n) & \xi(n-1) & \ldots & \xi(n-M+1)
\end{array}\right]^{T}
$$

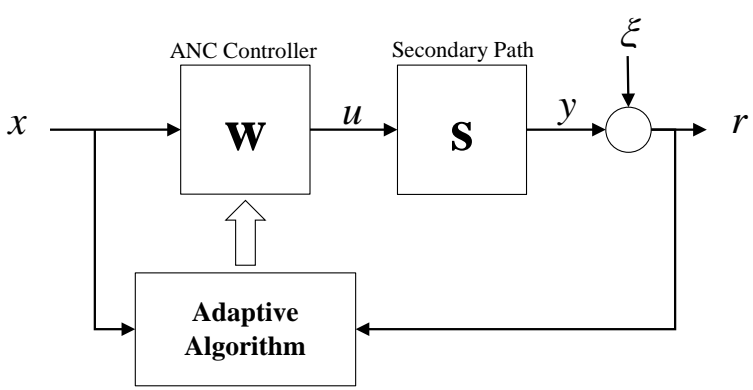

Figure 1: Block diagram of a generic ADAPTIVE ANC system

$$
\mathbf{y}=\left[\begin{array}{llll}
y(n) & y(n-1) & \ldots & y(n-M+1)
\end{array}\right]^{T}
$$

From Eq. (7), y can be formulated by

$$
\mathbf{y}=\mathbf{F}^{T} \mathbf{w}
$$

where $\mathbf{F}$ is a $L \times M$ matrix given by

$$
\mathbf{F}_{i j}=x_{s}(n-i-j-2), \quad i \in \mathbb{N} \leq L, \quad j \in \mathbb{N} \leq M
$$

Combining Eqs. (11) and (14) results in

$$
\mathbf{r}=\boldsymbol{\xi}+\mathbf{F}^{T} \mathbf{w}
$$

Let us assume that noise is a zero-mean Gaussian variable; thus,

$$
\pi(\boldsymbol{\xi}) \propto \exp \left(-\frac{1}{2 \sigma_{\xi}^{2}}\|\boldsymbol{\xi}\|^{2}\right)
$$

where $\pi(\boldsymbol{\xi})$ is the probability density function of $\boldsymbol{\xi}, \sigma_{\xi}^{2}$ is the variance of noise samples and $\|$.$\| denotes the Euclidean norm.$ For a given $\mathbf{w}$, the assumption of mutual independence of $\mathbf{w}$ and $\boldsymbol{\xi}$ ensure that probability density of $\boldsymbol{\xi}$ remains unaltered when conditioned on $\mathbf{w}$. Therefore,

$$
\begin{aligned}
\pi(\mathbf{r} \mid \mathbf{w}) & =\int \pi(\mathbf{r} \mid \mathbf{w}, \boldsymbol{\xi}) \pi(\boldsymbol{\xi}) d \boldsymbol{\xi} \\
& =\int \delta\left(\mathbf{r}-\boldsymbol{\xi}-\mathbf{F}^{T} \mathbf{w}\right) \pi(\boldsymbol{\xi}) d \boldsymbol{\xi} \\
& =\pi_{\xi}\left(\mathbf{r}-\mathbf{F}^{T} \mathbf{w}\right)
\end{aligned}
$$

Combining Eq. (17) and (18) results in

$$
\pi(\mathbf{r} \mid \mathbf{w}) \propto \exp \left(-\frac{1}{2 \sigma_{\xi}^{2}}\left\|\mathbf{r}-\mathbf{F}^{T} \mathbf{w}\right\|^{2}\right)
$$

The conditional probability density $\pi(\mathbf{w} \mid \mathbf{r})$ is considered as the posterior density of $\mathbf{w}$. This density expresses what is known about $\mathbf{w}$ after the realized observation $\mathbf{r}$. According to the Bayes formula,

$$
\pi_{\text {post }}(\mathbf{w})=\pi(\mathbf{w} \mid \mathbf{r})=\frac{\pi(\mathbf{r} \mid \mathbf{w}) \pi_{p r}(\mathbf{w})}{\pi(\mathbf{r})}
$$

where $\pi_{p r}(\mathbf{w})$ is the prior density of $\mathbf{w}$, describing what is known about $\mathbf{w}$ prior to the measurement. Here, a Gaussian prior density is assumed.

$$
\pi_{p r}(\mathbf{w}) \propto \exp \left(-\frac{1}{2 \sigma_{s}^{2}}\|\mathbf{w}-\overline{\mathbf{w}}\|^{2}\right)
$$

where $\overline{\mathbf{w}}$ and $\sigma_{w}^{2}$ are the mean and variance of the prior density. Substituting Eqs. (19) and 21 into 20 results in

$$
\pi_{\text {post }}(\mathbf{w}) \propto \exp \left(-\frac{1}{2 \sigma_{\xi}^{2}}\left\|\mathbf{r}-\mathbf{F}^{T} \mathbf{w}\right\|^{2}-\frac{1}{2 \sigma_{w}^{2}}\|\mathbf{w}-\overline{\mathbf{w}}\|^{2}\right)
$$


By maximizing $\pi_{\text {post }}(\mathbf{w})$, a Maximum A Posteriori (MAP) estimation for $\mathbf{w}$ can be calculated:

$$
\mathbf{w}_{M A P}=\arg \max _{\mathbf{w}} \pi(\mathbf{w} \mid \mathbf{r})
$$

Combining Eqs. (22) and (23) would lead to

$$
\mathbf{w}_{M A P}=\arg \max _{\mathbf{w}}\left\{\frac{1}{2 \sigma_{\xi}^{2}}\left\|\mathbf{r}-\mathbf{F}^{T} \mathbf{w}\right\|^{2}+\frac{1}{2 \sigma_{w}^{2}}\|\mathbf{w}-\overline{\mathbf{w}}\|^{2}\right\}
$$

Thus, $\mathbf{w}_{M A P}$ would be the solution of

$$
\nabla\left\{\frac{1}{2 \sigma_{\xi}^{2}}\left\|\mathbf{r}-\mathbf{F}^{T} \mathbf{w}\right\|^{2}+\frac{1}{2 \sigma_{w}^{2}}\|\mathbf{w}-\overline{\mathbf{w}}\|^{2}\right\}=0
$$

which is in the form of

$$
\mathbf{w}_{M A P}=\left(\mathbf{I}+\mu \mathbf{F F}^{T}\right)^{-1}(\overline{\mathbf{w}}-\mu \mathbf{F r})
$$

where $\mathbf{I}$ is the identity matrix of order $M$ and

$$
\mu=\frac{\sigma_{w}^{2}}{\sigma_{\xi}^{2}}
$$

Eq. (26) gives an optimal solution for the ANC filter; however, its realization is not computationally efficient. An adaptive algorithm for the realization of Eq. 26 is derived in the next section.

\section{An Adaptive Algorithm FOR Iterative CALCULATION OF $\mathbf{w}_{M A P}$}

Given that $\mathbf{w}_{M A P}(n-1)$ is the best guess for $\mathbf{w}$ at time index $n-1$, we would estimate $\mathbf{w}_{M A P}(n)$ as a maximum a posteriori estimate when conditioned on the most recent sample of the residual noise, $r(n)$ :

$$
\mathbf{w}(n)=\mathbf{w}_{M A P}(n)=\arg \max _{\mathbf{w}} \pi(\mathbf{w} \mid r(n)),
$$

which is a very especial case of Eq. (23) with $M=1$. In this situation, matrix $\mathbf{F}$ is simplified to a row vector in $\mathbb{R}^{L}$. We represent this vector by

$$
\mathbf{f}=\left[\begin{array}{llll}
x_{s}(n) & x_{s}(n-1) & \ldots & x_{s}(n-L+1)
\end{array}\right]^{T}
$$

Now, $\mathbf{w}(n)$ can be formulated by substituting $\overline{\mathbf{w}}=\mathbf{w}(n-1)$, $\mathbf{r}=r(n)$ and $\mathbf{F}=\mathbf{f}$ into Eq. 26.

$$
\mathbf{w}(n)=\left(\mathbf{I}+\mu \mathrm{ff}^{T}\right)^{-1}\{\mathbf{w}(n-1)-\mu \mathbf{f} r(n)\}
$$

The recursive algorithm given in Eq. 29, involves in a matrix inversion, which is not appropriate for implementation by using commercial real-time embedded systems. In the following, an alternative formulation which avoid matrix inversion is derived.

Infinite summation of geometric series of a matrix $\mathbf{M}$ converges to $(\mathbf{I}-\mathbf{M})^{-1}$ provided that all the eigenvalues of $\mathbf{M}$ are between -1 and 1 . Thus, by setting $\mathbf{M}=-\mu \mathrm{ff}^{T}$, one can deduce that

$$
\left(\mathbf{I}+\mu \mathbf{f f}^{T}\right)^{-1}=\sum_{k=0}^{\infty}\left(-\mu \mathbf{f f}^{T}\right)^{k}
$$

provided that all the eigenvalues of $\mu \mathrm{ff}^{T}$ are located between -1 and 1 . Matrix $\mathrm{ff}^{T}$ is a positive definite symmetric matrix that has $L-1$ zero eigenvalues and one non-zero eigenvalue of $\|\mathbf{f}\|^{2}$. Accordingly, $\mu \mathrm{ff}^{T}$ has $L-1$ zero eigenvalues and one non-zero eigenvalue of $\mu\|\mathbf{f}\|^{2}$. Therefore, the identity given in Eq. 30. only requires

$$
\mu\|\mathbf{f}\|^{2}<1 \Rightarrow \mu<\frac{1}{\|\mathbf{f}\|^{2}}
$$

For large $L,\|\mathbf{f}\|^{2}$ can be approximated by

$$
\|\mathbf{f}\|^{2}=L P_{x_{s}}
$$

where $P_{x_{s}}$ is the power of $x_{s}(n)$. Substituting this solution into Eq. (31) results in the following condition for the convergence of $\left(\mathbf{I}+\mu \mathrm{ff}^{T}\right)^{-1}$.

$$
\mu<\frac{1}{L P_{x_{s}}} .
$$

It is now desired to derive a closed-form expression for the right side of the identity given in Eq. (30). It can be initiated by calculating $\left(\mathrm{ff}^{T}\right)^{2}$ as follows.

$$
\left(\mathrm{ff}^{T}\right)^{2}=\left(\mathrm{ff}^{T}\right)\left(\mathrm{ff}^{T}\right)=\mathbf{f}\left(\mathbf{f}^{T} \mathbf{f}\right) \mathbf{f}^{T}
$$

Since $\mathbf{f}$ is a vector, $\mathbf{f}^{T} \mathbf{f}$ is a scalar and equal to the norm of $\mathbf{f}$, which can be estimated by $\mathrm{ff}^{T}=\|\mathbf{f}\|^{2}$. Substituting this result into Eq. (34) and using Eq. (32) results in

$$
\left(\mathrm{ff}^{T}\right)^{2} \approx L P_{x_{s}} \mathrm{ff}^{T}
$$

Multiplying both sides of this identity by $\mathrm{ff}^{\mathbf{T}}$ and replacing $\left(\mathrm{ff}^{T}\right)^{2}$ with $L P_{x_{s}} \mathrm{ff}^{T}$ results in

$$
\left(\mathrm{ff}^{T}\right)^{3} \approx L^{2} P_{x_{s}}^{2} \mathrm{ff}^{T}
$$

By repeating this process, the following general expression for the powers of $\mathrm{ff}^{\mathbf{T}}$ can be derived.

$$
\left(\mathbf{f f}^{\mathbf{T}}\right)^{k}=\left(L P_{x_{s}}\right)^{k-1} \mathbf{f f}^{\mathbf{T}}, \quad k \geq 1
$$

Substituting Eq. 37) into the right side of the Eq. 30 gives

$$
\left(\mathbf{I}+\mu \mathbf{f f}^{T}\right)^{-1}=\mathbf{I}+\frac{1}{L P_{x_{s}}} \sum_{k=1}^{\infty}\left(-\mu L P_{x_{s}}\right)^{k} \mathbf{f f}^{\mathbf{T}}
$$

which can be simplified by

$$
\begin{aligned}
\left(\mathbf{I}+\sigma_{w / \xi}^{2} \mathbf{f f}^{T}\right)^{-1} & =\mathbf{I}-\frac{\mu}{1+\mu L P_{x_{s}}} \mathbf{f f}^{T} \\
& =\mathbf{I}-\mu \mathrm{ff}^{T}
\end{aligned}
$$

where $\mu$ is defined by

$$
\rho=\frac{\mu}{1+\mu L P_{x_{s}}}
$$

Now, substituting Eq. 39] into 29, results in

$$
\mathbf{w}(n)=\left(\mathbf{I}-\rho \mathbf{f f}^{T}\right)(\mathbf{w}(n-1)-\mu \mathbf{f} r(n))
$$

which is the proposed iterative algorithm for the maximum a posteriori estimation of ANC filters. 

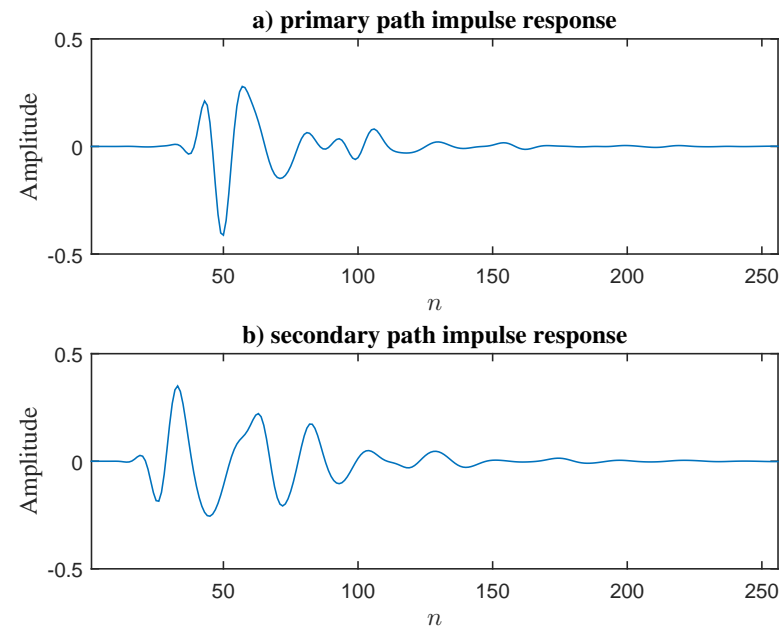

Figure 2: Impulse responses of the primary and secondary paths used in computer simulation

Derivation of the FxLMS algorithm as a special case of the proposed algorithm

For a large $L$ and a Gaussian white noise, one can conclude

$$
\mathrm{ff}^{T} \approx P_{x_{s}} \mathbf{I}
$$

In this case, $\mathbf{I}-\rho \mathrm{ff}^{T}$ is simplified to

$$
\begin{aligned}
\mathbf{I}-\rho \mathbf{f f}^{T} & =\mathbf{I}-\frac{\mu}{1+\mu L P_{x_{s}}} \mathbf{f f}^{T} \\
& =\left(1-\frac{\mu P_{x_{s}}}{1+\mu L P_{x_{s}}}\right) \mathbf{I} \\
& =\left(\frac{1+\mu(L-1) P_{x_{s}}}{1+\mu L P_{x_{s}}}\right) \mathbf{I}
\end{aligned}
$$

For large $L$ (high order adaptive filters),

$$
\frac{1+\mu(L-1) P_{x_{s}}}{1+\mu L P_{x_{s}}} \approx 1
$$

Thus,

$$
\mathbf{I}-\rho \mathrm{ff}^{T} \approx \mathbf{I}
$$

Substituting this result into Eq. 41 results in

$$
\mathbf{w}(n)=\mathbf{w}(n-1)-\mu \mathbf{f} r(n)
$$

which is identical to the well-known FxLMS algorithm. In fact, the FxLMS algorithm is a very special case of the proposed algorithm, where the noise to be canceled is a Gaussian white process and the adaptive filter length is high.

\section{Computer Simulation}

This section demonstrates the validity of the theoretical results by using computer simulation. The impulse responses of the primary and secondary paths used in the computer simulations are shown in Figure 2. The impulse responses are measured in an experimental ANC setup installed in an acoustic duct [12]. The acoustic duct and experimental setup is discussed in the next section. For simulating the system
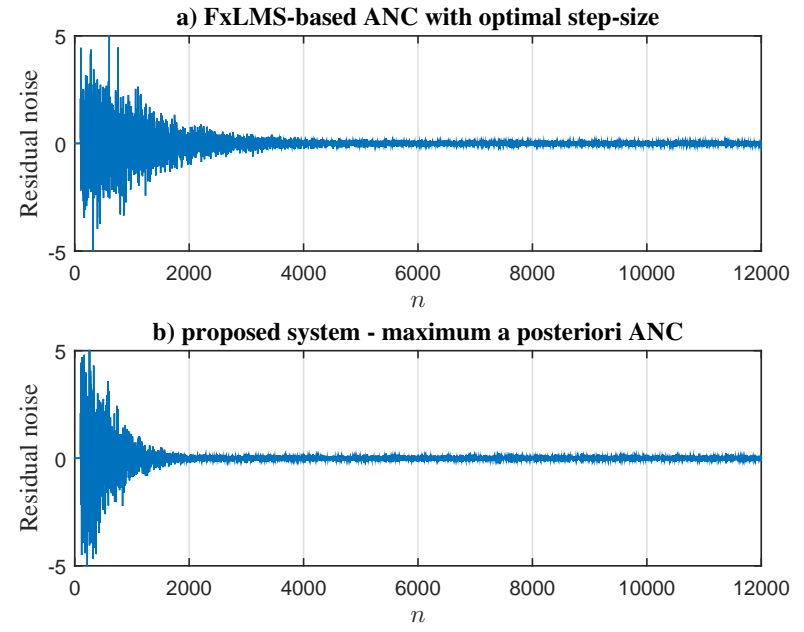

Figure 3: Residual noise signal obtained by using FxLMS algorithm and the proposed algorithm for white noise

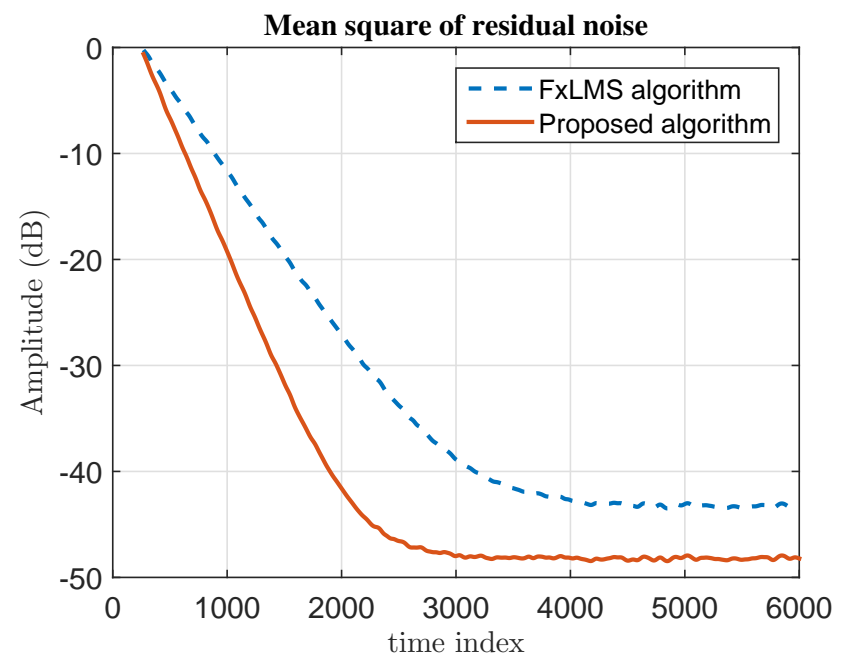

Figure 4: Mean square of the residual noise obtained by using FxLMS algorithm and the proposed algorithm for white noise

operation, two MATLAB codes are developed: one for an FxLMS-based ANC system and one for the proposed ANC system.

In the first computer simulation experiment, a white signal with the power of 1 is used as the original noise. In this case, both the FxLMS-based and proposed ANC systems can successfully attenuate the noise. Figure 3 shows the variation of the residual noise obtained by using the two ANC systems. The step-size of the FxLMS algorithm is set to its optimal level as proposed in [16]. It can be seen visually, that the convergence rate of the proposed ANC system is higher than that of the FxLMS-based ANC system. This experiment is repeated for 500 times with independent random noise signals. The residual noise signal for each experiment run is recorded and the mean square of the residual noise is calculated by averaging over the square of the residual noise signals. This measure is usually called Mean Square Error (MSE) in ANC 

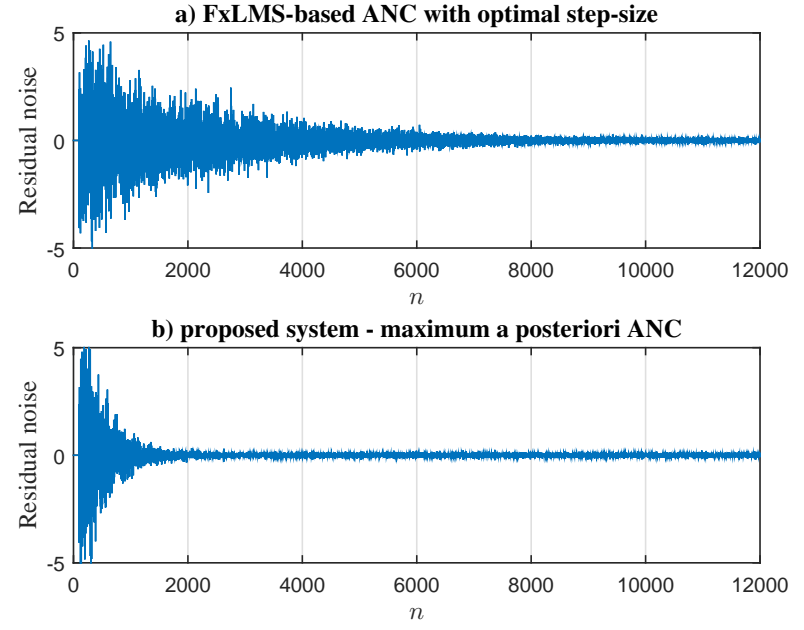

Figure 5: Residual noise signal obtained by using FxLMS algorithm and the proposed algorithm for pink noise

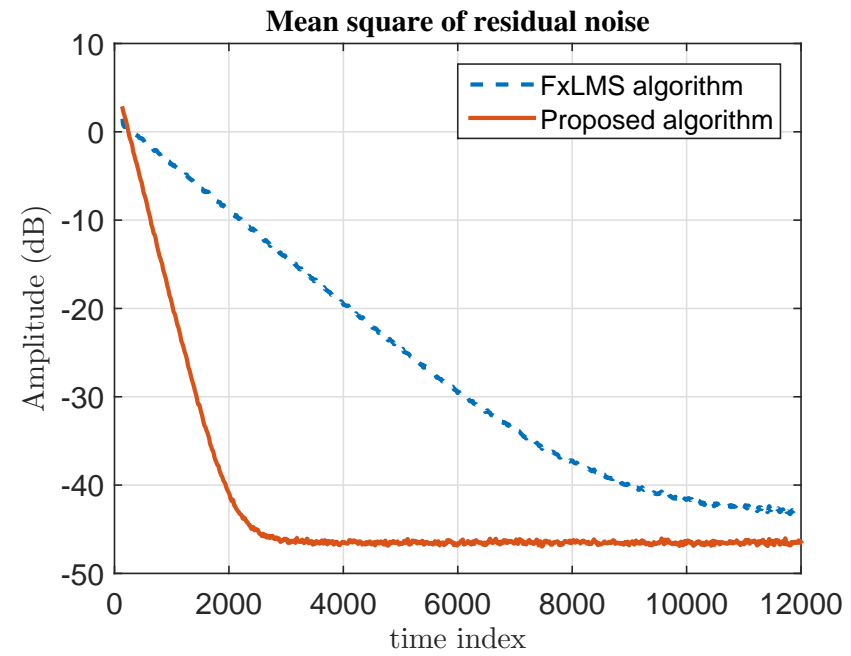

Figure 6: Mean square of the residual noise obtained by using FxLMS algorithm and the proposed algorithm for pink noise

literature, representing the performance of the adaptation process. The convergence rate of the MSE signal shows the speed of the adaptation process performed by the adaptive algorithm and the steady-state level of it shows the residual noise level in steady-state conditions. The MSE curves obtained by using the FxLMS-based and proposed ANC systems are shown in Figure 4. As seen, the performance of the proposed ANC system is higher than that of the FxLMS-based ANC in terms of convergence rate and steady-state level.

In the second experiment, a pink noise with the power 1 is used as the original noise and the same procedure discussed above is performed. The variation of the residual noise is shown in Figure 5 and the MSE function is shown in Figure 6 As seen, the proposed system has a higher performance in terms of the convergence rate and steady-state performance, similar to the previous experiment. It can be also concluded from the above experiments that the preference of the proposed ANC system to the traditional FxLMS-based ANC system is more evident when the noise to be canceled is not a white process.

\section{EXPERIMENTAL RESULTS}

This section reports a successful implementation of the proposed ANC system. The key hardware component used for the implementation of the system is a Single Board RIO 9627 (sbRIO-9627) manufactured by National Instruments. This processor has a $667 \mathrm{MHz}$ dual-core ARM Cortex-A9 based microprocessor and a reconfigurable FPGA chip (Xilinx Zynq7000 XC7Z020 All Programmable SoC) on which the code of the proposed algorithm is executed. C series modules NI 9234E and NI 9263 are used for acoustic signal acquisition. NI 9234E module is a compact analogue input module which features 4 channel 24-bit resolution ADC and whose input range is $-5 \mathrm{~V}$ to $5 \mathrm{~V}$. It can accept either differential input or single-end input signal. NI 9263 module is a powerful analogue output featuring 16-bit resolution DAC and whose output range is $-10 \mathrm{~V}$ to $10 \mathrm{~V}$. The microphones used for picking up residual noise signal (error microphone) and reference signal (reference microphone) are dynamic cardioid microphones (AKG 770D) with the sensitivity of $-52 \mathrm{~dB}(2.5 \mathrm{mV} / \mathrm{Pa})$. The microphones have a flat frequency response at low frequency range. The loudspeaker used as the anti-noise source is CW2190 with the rated output power of 27 watts and impedance of $8 \mathrm{ohms}$; the self-designed power amplifier producing 15 watts at $8 \mathrm{ohms}$ is used to drive the loudspeaker.

The control system is installed in an acoustic duct with the dimensions of $1600 \mathrm{~cm} \times 22 \mathrm{~cm} \times 33 \mathrm{~cm}$. A random noise (band-limited white noise with the power of $20 \mathrm{~dB}$ and frequency range of $300-1000 \mathrm{~Hz}$ ) is generated by a computer and injected into the opening of the duct as shown in Figure 7 The location of the canceling loudspeaker and microphones are also shown in Figure 7 The sampling frequency of the control system is set to $6 \mathrm{KHz}$. A secondary path model is measured by using off-line secondary path modeling technique prior to the operation of the ANC system [15]. The two parameters of the adaptive algorithm are set to $\mu=0.002$ and $\rho=2 e-5$. Figure 8 shows the results obtained from the experiment. Figure $8 \mathrm{a}$ shows the variation of the residual noise. As seen, the residual noise power converges to its steady-state level of $-3 \mathrm{~dB}$ after about 0.5 Seconds. Figure $8 \mathrm{a}$ compares the spectra of the original noise and residual noise. As seen, the proposed ANC system can efficiently attenuate the noise over its frequency range.

\section{CONCLUSION}

Bayesian statistics provide a very reliable platform for the derivation of real-time signal processing algorithms. A realtime adaptive algorithm for the adjustment of transversal adaptive controllers (filters) used in active noise control is proposed in this paper. The joint probability desnity distribution of the ANC filter parameters are formulated and its maximum a posteriori solution is calculated to be used as the optimal solution for the ANC filter parameters. This optimal solution for the parameters of the adaptive controller is given in terms of available signals, i.e. reference signal and 


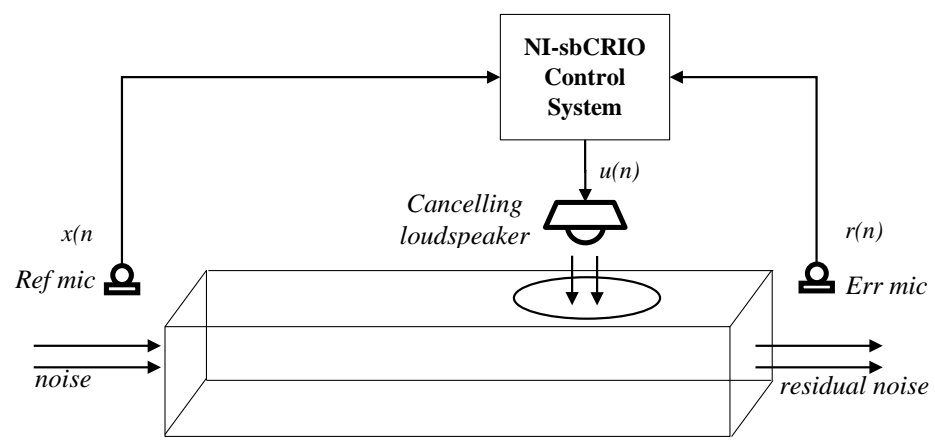

Figure 7: Schematic diagram of the experimental setup

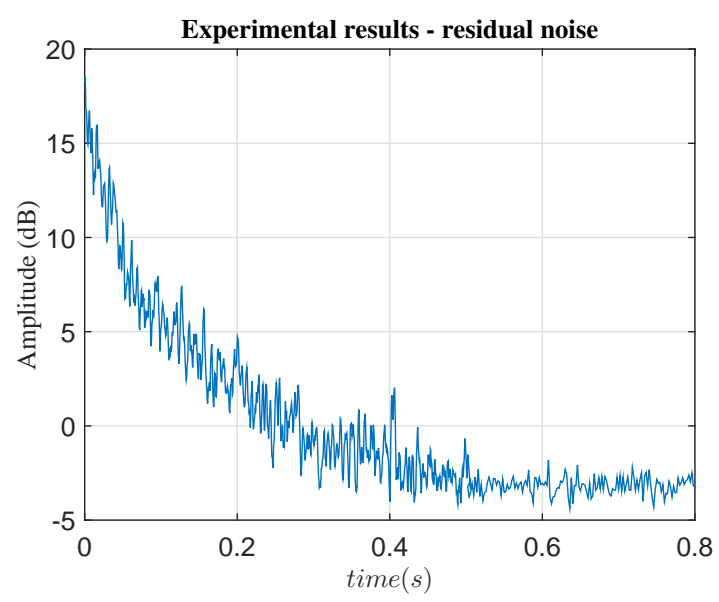

(a) Variation of the residual noise

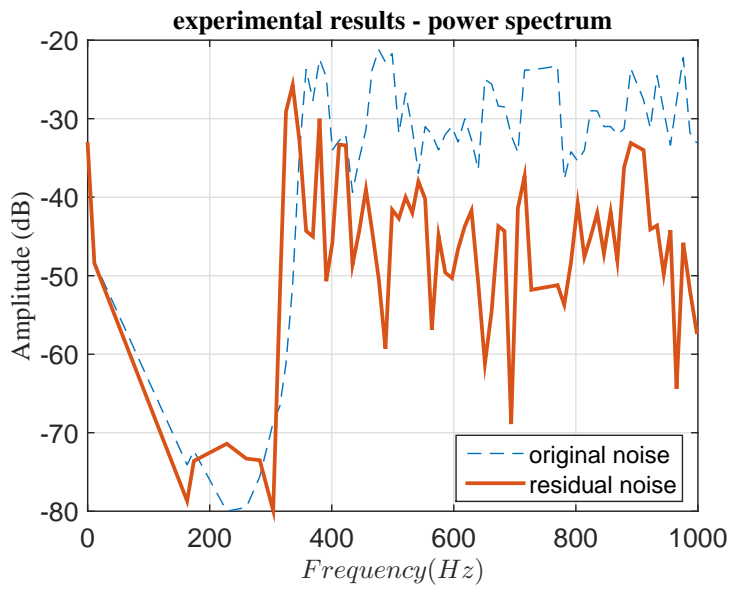

(b) Spectra of the original and residual noise

Figure 8: Experimental results

residual noise. Real-time implementation of this solution is not computationally efficient. Hence, a simple iterative algorithm is developed to reach an estimate of the optimal solution. The proposed algorithm is found efficient in both computer simulation and practical experiments.

\section{REFERENCES}

[1] C. H. Hansen, Understanding Active Noise Cancellation, 1st ed. London EC4P 4EE, UK: Taylor \& Francis Group, 2001, ch. 2.
[2] S. Haykin, Adaptive Filter Theory, 4th ed. Prentice Hall, 2002, ch. 9, 13.

[3] S. M. Kuo and D. R. Morgan, Active noise control systems: algorithms and DSP implementations. New York, NY, USA: Wiley Interscience, 1996.

[4] J. Kaipio and E. Somersalo, Statistical and Computational Inverse Problems. Springer-Verlag New York, 2005, ch. 3.

[5] G. E. Warnaka, L. A. Poole, and J. Tichy, "Active acoustic attenuators," U.S. Patent 4473906, 1984.

[6] J. C. Burgess, "Active adaptive sound control in a duct: computer simulation," Journal of the Acoustical Society of America, vol. 70, pp. 715-726, 1981.

[7] B. Widrow, D. Shur, and S. Shaffer, "On adaptive inverse control," in Proceeding of the 15th Asilomar Conference on Circuits, Systems and Computers, Nov. 1981, pp. 185-189.

[8] I. Tabatabaei Ardekani and W. H. Abdulla, "FxLMS-based active noise control: a quick review," Proceedings of 2011 Asia Pacific Signal and Information Processing Association Annual (APSIPA) Summit and Conference, Xi'an, China, 2011.

[9] L. J. Eriksson and M. C. Allie, "Use of random noise for online transducer modeling in an adaptive active attenuation system," The Journal of the Acoustical Society of America, vol. 85, no. 2, pp. 797802, 1989.

[10] M. Zhang, H. Lan, and W. Ser, "Cross-updated active noise control system with online secondary path modeling," IEEE Transactions on Speech and Audio Processing, vol. 9, no. 5, pp. 598 -602, Jul. 2001.

[11] M. T. Akhtar, M. Abe, and M. Kawamata, "A new structure for feedforward active noise control systems with improved online secondary path modeling," IEEE Transactions on Speech and Audio Processing, vol. 13 , no. 5, pp. 1082-1088, Sep. 2005.

[12] I. T. Ardekani, J. P. Kaipio, A. Nasiri, H. Sharifzadeh, and W. H Abdulla, "A statistical inverse problem approach to online secondary path modeling in active noise control," IEEE/ACM Transactions on Audio, Speech, and Language Processing, vol. 24, no. 1, pp. 54-64, Jan 2016.

[13] I. Tabatabaei Ardekani and W. H. Abdulla, "On the convergence of realtime active noise control systems," Signal Processing, vol. 91, no. 5, pp. 1262-1274, 2011.

[14] I. T. Ardekani, H. Sharifzadeh, S. Rehman, and W. H. Abdulla, "Efficient fxlms algorithm with simplified secondary path models," in 2015 IEEE International Conference on Acoustics, Speech and Signal Processing (ICASSP), April 2015, pp. 609-613.

[15] I. Tabatabaei Ardekani and W. H. Abdulla, "Effects of imperfect secondary path modeling on adaptive active noise control systems," IEEE Transactions on Control Systems Technology, vol. 20, no. 5, pp. $1252-1262$, sept. 2012.

[16] I. Tabatabaei Ardekani and W. Abdulla, "Stochastic modelling and analysis of filtered-x least-mean-square adaptation algorithm," Signal Processing, IET, vol. 7, no. 6, pp. 486-496, August 2013. 